\title{
Percepción y uso de mamíferos no voladores en las localidades de Aguas Blancas y el Chimborazo en la Subregión Costanera del departamento de Córdoba, Colombia
}

\author{
Julio Chacón Pacheco \\ Grupo de Investigación Biodiversidad Unicórdoba, Universidad de Córdoba, Montería, Córdoba, Colombia; jchacon_bio@hotmail.com \\ Juan Camilo Salcedo López \\ Licenciado en Ciencias Naturales y Educación Ambiental, Universidad de Córdoba, Montería, Córdoba, Colombia
}

El conocimiento tradicional, entendido como las sabidurías y conocimientos colectivos e integrales de las comunidades fundamentadas en la práctica y su interacción con el medio natural, refleja cómo las poblaciones humanas se relacionan con el entorno (Machado-Trelles, 2008; De la Cruz 2010). Áreas del conocimiento como las etnociencias buscan entender el mundo como es percibido, abordando conceptos relacionados a la relación del hombre con la biodiversidad, entre estas, la etnozoología propone conocer sobre la relación fauna-hombre (Grebe, 1984). Los animales silvestres han sido de primordial importancia para la especie humana al ser utilizados en diversos aspectos, como fuente de alimentación, en la elaboración de diversos utensilios, la domesticación, el simbolismo mitico-religioso, entre otros (Redford \& Robinson, 1991; Ojasti, 2000; Robinson \& Bennett, 2000). En este sentido, los animales se han convertido en una de las imágenes más poderosas para el ser humano, tanto en el mundo externo como en el interno (Ramos et al., 2005). Las relaciones y actitudes que desarrollan los seres humanos hacia los animales, dependen de factores relacionados directamente con el hombre como lo son la cultura, la historia, la sociedad, la economía y el pensamiento individual; igualmente estos factores también están relacionados a las condiciones naturales de las especies como es la ecología, comportamiento, distribución, epidemiología, entre otros (Turbay 2002).

De acuerdo a esto, las relaciones que se dan entre humano - naturaleza, permiten comprender la percepción y uso de la fauna, así como las problemáticas puntuales que pueden afectar la fauna silvestre en las localidades de estudio, como lo son la cacería, el tráfico ilegal y la transformación de los hábitats. De modo que, la percepción se entiende como la idea que representa el hombre dependiendo del ordenamiento, y clasificación de estímulos desde una cosmovisión articulada en un contexto social, cultural e histórico (Ceballos-Mago \& Chivers, 2010). Además, nos permite conocer cuáles son los patrones de uso de la mastofauna como recurso de valor económico y tradicional (Racero-Casarrubia et al., 2008; Cunha-Ribeiro \& Schiavetty, 2009).

El departamento de Córdoba es considerado un eje importante en la comercialización y uso de fauna silvestre (Carrascal et al., 2013; Humanez et al., 2015; Chacón et al. en prensa). Sin embargo, todavía es posible encontrar vacíos en las investigaciones que buscan reconocer la percepción y uso por parte de los pobladores en las distintas subregiones del Departamento. En la actualidad se reconocen trabajos realizados para las subregiones de Alto Sinú, Sinú Medio y San Jorge, donde se ha buscado conocer los patrones de comercialización de fauna silvestre en los mercados públicos, así como su uso y tráfico ilegal (Hoyos \& González, 2003; Ayazo, 2006; Racero-Casarrubia et al., 2008; Rhenals, 2010; Racero-Casarrubia \& González-Maya, 2014). Sin embargo, el estudio de los mamíferos para la subregión Costanera es escaso, registrando sólo el trabajo de Ballesteros (2015) en murciélagos asociados a sistemas de ganadería.

Es por esto, que el presente trabajo buscó conocer la percepción, amenazas y el uso de las especies de mamíferos no voladores, que le dan las comunidades campesi nas en las local idades de Aguas Blancas ( $8^{\circ} 47^{\prime} 11.8^{\prime \prime} \mathrm{N}-76^{\circ} 18^{\prime} 17.6^{\prime \prime} \mathrm{W}$ a $94 \mathrm{msnm}$ ) en el municipio de Los Córdobas y El Chimborazo ( $8^{\circ} 44^{\prime} 34.6^{\prime \prime}$ - 76¹9'28.3"W a 89 msnm) ubicado en el municipio de Canalete, Córdoba, Colombia (Figura 1). Estas localidades presentan parches de bosque seco tropical (bs-T) aislados en estadio secundario, rodeados por una matriz de ganadería extensiva convencional ligada a grandes terratenientes, lo que afecta a las familias campesinas, que soportan su economía con pocas áreas de cultivo, la pesca y la extracción de fauna silvestre para suplir las necesidades básicas de alimentación. Estas zonas, poseen un clima cálido tropical, temperatura promedio de

\footnotetext{
Mammalogy Notes | Notas Mastozoológicas

Sociedad Colombiana de Mastozoología

Vol. 4 Num. 1| 2017
} 
$27.8^{\circ} \mathrm{C}$, precipitación unimodal con un promedio anual de $1300 \mathrm{~mm} / \mathrm{año}$, con una época seca de diciembre-marzo, y una época de lluvias de abril-noviembre (Palencia et al., 2006).

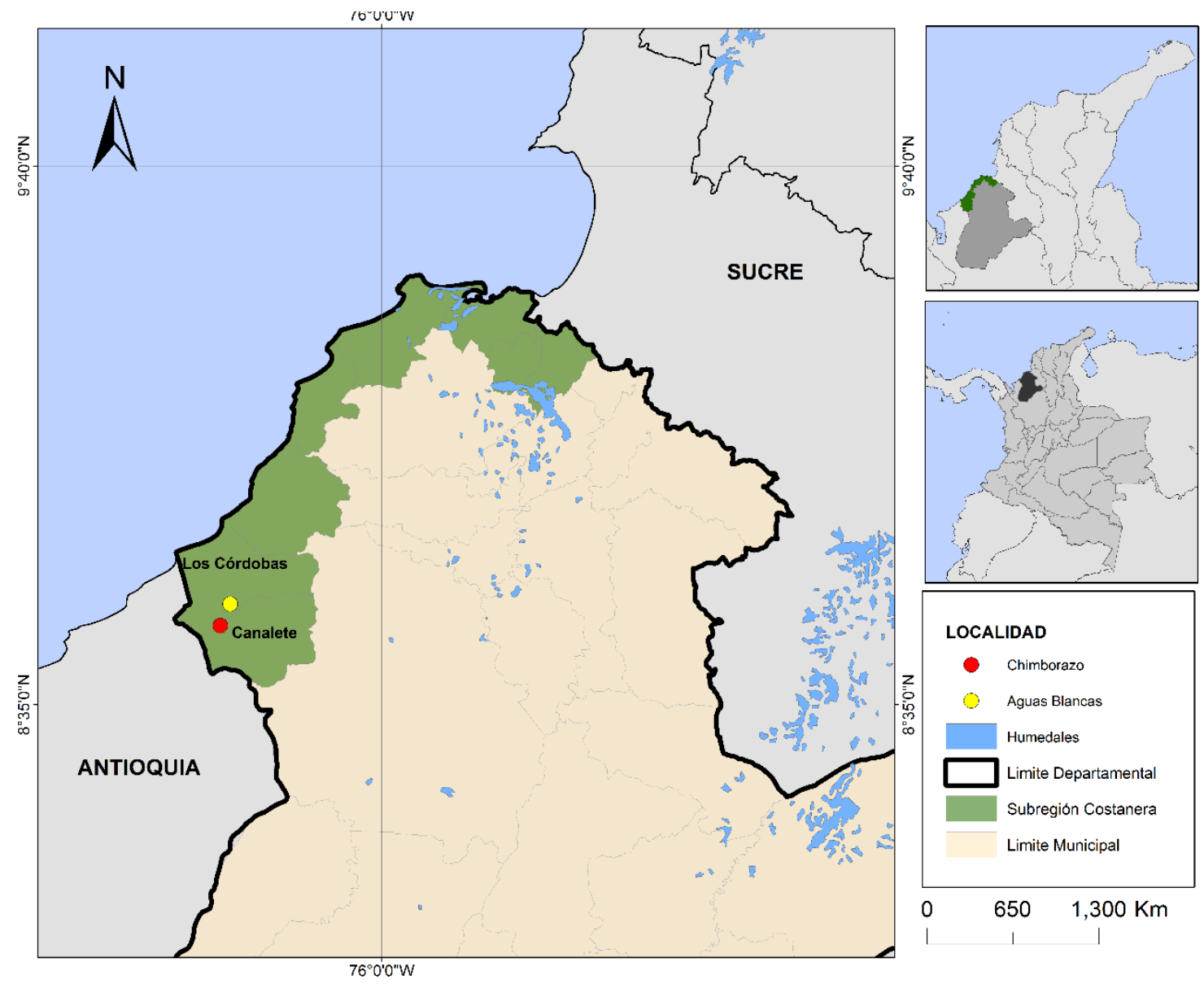

Figura 1. Ubicación geográfica de las localidades de Aguas Blancas y El Chimborazo, Subregión Costanera del departamento de Córdoba, Colombia.

El listado de mamíferos, su uso y las amenazas se obtuvieron a partir de entrevistas y talleres con los pobladores entre los meses de febrero a mayo de 2016, empleando guías de campo ilustradas (Tirira, 2007; Chacón \& Humanez, 2012). Para las localidades de Aguas Blanca y El Chimborazo se pudieron entrevistar 71 y 67 personas respectivamente.

Los pobladores identificaron 22 especies de mamíferos no voladores pertenecientes a 20 familias y ocho órdenes (Tabla 1), lo que representa el 18.8\% de los mamíferos presentes (117 especies) en el departamento de Córdoba (Racero-Casarrubia et al., 2015). La mayor riqueza de especies la presenta el orden Carnivora (6 especies) con las familias Felidae y Procyonidae.

Los usos que se registraron corresponden al consumo, comercio, uso como mascotas y el ornamental. Para el caso del consumo de carne de monte se registraron 13 especies de mamíferos no voladores seguida del comercio y el uso ornamental con cinco especies cada uno, y por último, el uso como mascotas con cuatro especies de las cuales tres son primates (Alouatta seniculus, Cebus capucinus y Saguinus oedipus) (Tabla 1, Figura 2). Los pobladores destacan la importancia de especies como el armadillo (Dasypus novemcinctus), el ñeque (Dasyprocta puntacta), la guartinaja (Cuniculus paca) y el conejo (Silvilagus sp.), entre otras, como importantes porque les permite suplir sus necesidades básicas de alimentación gracias al aporte proteico.

\section{Mammalogy Notes | Notas Mastozoológicas \\ Sociedad Colombiana de Mastozoología \\ Vol. 4 Num. 1| 2017}




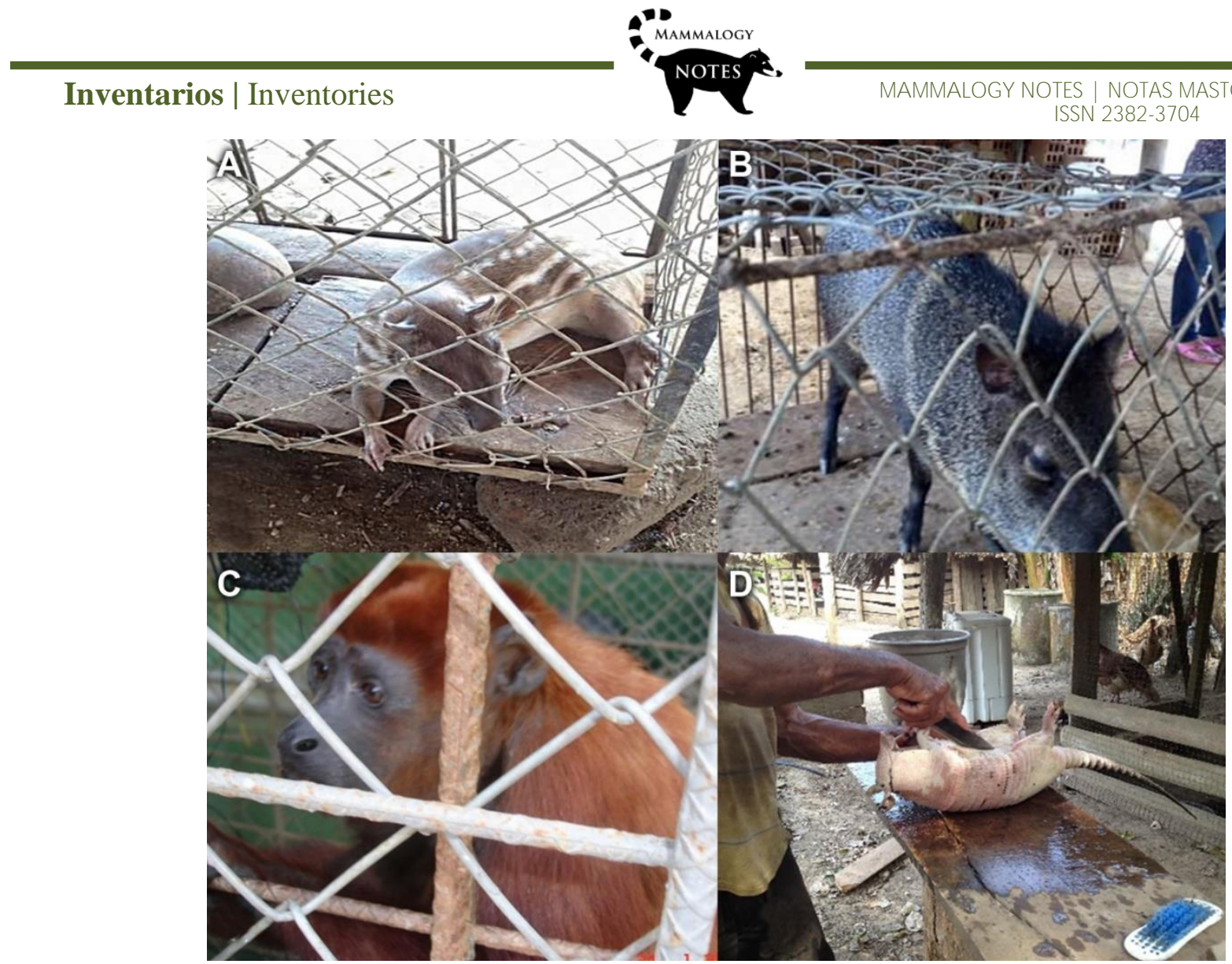

Figura 2. Registro fotográfico de las especies presentes y de uso en las localidades de Aguas Blancas y Chimborazo en los municipios de Los Córdobas y Canalete, Córdoba, Colombia. A) Guartinaja (Cuniculus paca) en cautiverio, B) Zaino (Pecari tajacu) en cautiverio, C) Mono colorado (Alouatta seniculus) en cautiverio, D) preparación de armadillo (Dasypus novemcinctus).

En la localidad de El Chimborazo no se evidencia la comercialización de fauna silvestre, debido a que las especies se encuentran protegidas por los dueños de predios con fragmentos de bosque, actuando como guardabosques. Sin embargo, se presenta la cacería de control de especies que pueden generar daños a cultivos y especies domésticas, como es el caso de la zorra chucha (Didelphis marsupialis) y algunos carnívoros, como son la zorra baya (Cerdocyon thous) y la zorra tejón (Procyon cancrivorus).

Las amenazas identificadas por los pobladores que más impacto generan sobre los mamíferos son: la cacería que afecta el $63.6 \%$ de las especies registradas, seguida de la ganadería (54.4\%) y la expansión agrícola (50\%). Lo cual, según las personas entrevistadas, ha llevado a la desaparición de especies como el jaguar (Panthera onca). Los pobladores afirman que especies consideradas abundantes hace aproximadamente 50 años, en la actualidad se consideran escasos, como es el caso del venado (Odocoileus caricou), el titi cabeciblanco (S. oedipus), el machín (C. capucinus), la marteja (Aotus sp.), la nutría (Lontra longicaudis), la guartinaja (C. paca), el ñeque (D. punctata), el hormiguero (Tamandua mexicana), el tigrillo (Leopardus pardalis), el perezoso de tres uñas (Bradypus variegatus) y el zaino (Pecari tajacu).

Los mamíferos de las localidades estudiadas juegan un papel importante en los aspectos sociales y económicos de los pobladores de estas localidades al estar involucrados en la dieta o en la generación de efectos negativos sobre cultivos y/o fauna doméstica. Pese a las problemáticas ambientales manifestadas, como la cacería ilegal y la tala de árboles, aún se encuentran parches de bs-T que albergan la diversidad de mamíferos registrada. Por lo tanto, es necesario generar acciones de protección y manejo para brindar la posibilidad de conservar un remanente de la fauna silvestre del departamento de Córdoba, reconociendo las problemáticas sociales, pobreza e inequidad social, que empujan a utilizar los mamíferos silvestres como una alternativa de proteína animal (Baptiste-Ballera et al., 2002; Racero-Casarrubia \& González-Maya, 2014). 
Tabla 1. Mamíferos registrados en Aguas Blancas y Chimborazo, municipios de Los Córdobas y Canalete, departamento de Córdoba, Colombia. Usos: C, Consumo; Co, Comercio; M, Mascota; O, Ornamental.

\begin{tabular}{|c|c|c|c|c|c|c|}
\hline \multirow[b]{2}{*}{ Orden } & \multirow[b]{2}{*}{ Familia } & \multirow[b]{2}{*}{ Especie } & \multirow[b]{2}{*}{ Nombre común } & \multicolumn{2}{|c|}{ Localidad } & \multirow[b]{2}{*}{ Uso } \\
\hline & & & & $\begin{array}{c}\text { Aguas } \\
\text { Blancas } \\
\end{array}$ & Chimborazo & \\
\hline \multirow{2}{*}{ Artiodactyla } & Cervidae & Odocoileus cariacou & Venado cola blanca & $\mathrm{X}$ & $\mathrm{X}$ & $\mathrm{C}, \mathrm{O}$ \\
\hline & Tayassuidae & Pecari tajacu & Zaino & $\mathrm{X}$ & & $\mathrm{C}$ \\
\hline \multirow{6}{*}{ Carnivora } & Canidae & Cerdocyon thous & Zorra baya & $\mathrm{X}$ & $\mathrm{X}$ & \\
\hline & Felidae & Leopardus pardalis & Tigrillo & $\mathrm{X}$ & $\mathrm{X}$ & $\mathrm{C}, \mathrm{O}, \mathrm{Co}$ \\
\hline & Felidae & Panthera onca & Tigre o Jaguar & $\mathrm{X}$ & & $\mathrm{C}, \mathrm{Co}, \mathrm{M}, \mathrm{O}$ \\
\hline & Mustelidae & Lontra longicaudis & Nutria & & $\mathrm{X}$ & \\
\hline & Procyonidae & Procyon cancrivorus & Zorra tejón & $\mathrm{X}$ & & \\
\hline & Procyonidae & Potos flavus & Leoncillo & $\mathrm{X}$ & & \\
\hline Cingulata & Dasypodidae & Dasypus novemcinctus & Armadillo & $\mathrm{X}$ & $\mathrm{X}$ & $\mathrm{C}$ \\
\hline Didelphiomorphia & Didelphidae & Didelphis marsupialis & Zorra chucha & $\mathrm{X}$ & $\mathrm{X}$ & \\
\hline Lagomorpha & Leporidae & Silvilagus sp & Conejo & $\mathrm{X}$ & $\mathrm{X}$ & $\mathrm{C}, \mathrm{Co}, \mathrm{O}$ \\
\hline \multirow{2}{*}{ Pilosa } & Bradypodidae & Bradypus variegatus & Oso perezoso & $\mathrm{X}$ & $\mathrm{X}$ & \\
\hline & Myrmecophagidae & Tamandua mexicana & Oso hormiguero & $\mathrm{X}$ & $\mathrm{X}$ & \\
\hline \multirow{4}{*}{ Primate } & Aotidae & Aotus sp & Marta & $\mathrm{X}$ & $\mathrm{X}$ & \\
\hline & Atelidae & Alouatta seniculus & Mono colorado & $\mathrm{X}$ & & $\mathrm{C}, \mathrm{M}$ \\
\hline & Callitrichidae & Saguinus oedipus & Titi cabeciblanco & $\mathrm{X}$ & $\mathrm{X}$ & $\mathrm{Co}, \mathrm{M}$ \\
\hline & Cebidae & Cebus capucinus & Machín & $\mathrm{X}$ & $\mathrm{X}$ & $\mathrm{C}, \mathrm{M}$ \\
\hline \multirow{5}{*}{ Rodentia } & Caviidae & Hydrochoerus isthmius & Chigüiro & & $\mathrm{X}$ & $\mathrm{C}$ \\
\hline & Cuniculidae & Cuniculus paca & Guartinaja & & $\mathrm{X}$ & $\mathrm{C}$ \\
\hline & Dasyproctidae & Dasyprocta punctata & Neque & $X$ & & $\mathrm{C}$ \\
\hline & Erethizontidae & Coendou sp & Puerco espino & $\mathrm{X}$ & & $\mathrm{C}, \mathrm{Co}$ \\
\hline & Sciuridae & Notosciurus granatensis & Ardilla & $\mathrm{X}$ & $\mathrm{X}$ & $\mathrm{C}, \mathrm{O}$ \\
\hline
\end{tabular}

Agradecimientos

A la Fundación Flora y Fauna y su director Alejandro Hoyos Begambre por su apoyo logístico. A Gustavo Cogollo por su acompañamiento en la elaboración de las charlas y encuestas. A las comunidades de las localidades de El Chimborazo y Aguas Blancas en el municipio de Canalete, departamento de Córdoba.

\section{Referencias}

AYAZO, R. 2006. Reconocimiento de la fauna silvestre comercializada en los mercados públicos de cinco municipios de la subregión del Sinú medio, córdoba, Colombia. Trabajo de Grado, Facultad Ciencias Básicas, Universidad de Córdoba, Montería, Colombia.

BALLESTEROS, J.C. 2015. Efecto del manejo silvopastoril y convencional de ganadería extensiva sobre el ensamblaje de murciélagos asociados a fragmentos de bosque seco tropical en Córdoba, Colombia. Tesis doctoral. Facultad Ciencias, Pontificia Universidad Javeriana, Bogotá, Colombia.

BAPTISTE-BALLERA, L.G., HERNÁNDEZ, S., POLANCO, R., \& QUICENO, M. 2002. La fauna silvestre colombiana: una historia económica y social de un proceso de marginalización. Pp. 295-340 en: Rostros culturales de la fauna (Ulloa, A. ed.). Instituto Colombiano de Antropología e Historia, Fundación Natura.

CARRASCAL, J., CHACÓN, J. \& OCHOA, V. 2013. Ingreso de psittacidos al centro de atención de fauna (CAV-CVS), durante los años 2007-2009. Revista MVZ Córdoba 18(1): 3414-3419.

CEBALLOS-MAGO, N. \& CHIVERS, D.J. 2010. Local knowledge and perceptions of per primates and wild Margarita capuchins on Isla de Margarita and Isla de Coche in Venezuela. Endangered Species Research 13: 63-72.

CHACÓN, J. \& HUMANEZ E. 2012. Guía Rápida á Colores de los mamíferos no voladores comunes en el Departamento de Córdoba, Colombia \#1. Enviromental \& Conservation programs, The Field Museum, Chicago, USA USA [http://fieldmuseum.org/IDtools].

CHACÓN, J., HUMANEZ-LÓPEZ E., GUERRA-GALVÁN L. \& CARRASCAL JC. En prensa. Diagnóstico del tráfico ilegal de primates en el departamento de Córdoba, Colombia. Revista Latinoamericana de Conservación.

CUNHA-RIBEIRO, G. \& SCHIAVETTY, A. 2009.-Conocimiento, creencias y utilización de la mastofauna por los pobladores del Parque Estatal de la Sierra de Conduru, Bahía, Brasil. (En) Costa-Neto, E.M., Santos-Fita, D. \& Vargas-Clavijo, M. (coord.) 2009. Manual de etnozoología. Una guía teóricopráctica para investigar la interconexión del ser humano con los animales. Ediciones tundra.

DE LA CRUZ, R. 2010. Conocimientos tradicionales, biodiversidad y derechos de propiedad intelectual-Patentes. Revista AFESE 54: 77-96.

GREBE, M.E. 1984. Etnozoología andina: concepciones e interacciones del hombre andino con la fauna altiplánica. Estudios Atacameños 7: $335-347$.

HUMANEZ-LÓPEZ, E., CHACON, J., \& PLESE, T. 2015. Áreas de extracción de Xenartros en el Caribe colombiano. Edentata 16: 65-68

MACHADO-TRELLES, Y. 2008. Los saberes tradicionales populares. Un acercamiento desde el Interaccionismo Simbólico 25-35. (En) Red Gestcon. Gestión del Conocimiento Tradicional. Experiencias desde la Red GESTCON. Ed. Gente Nueva Editores.

OJASTI, J. 2000. Manejo de Fauna Silvestre Neotropical. SIMAB Series N 5. Smithsonian Institution / MAB Program. Washington, D. C.

\section{Mammalogy Notes | Notas Mastozoológicas \\ Sociedad Colombiana de Mastozoología \\ Vol. 4 Num. 1| 2017}

